\title{
Gauged double field theory
}

\section{Mariana Graña and Diego Marques}

Institut de Physique Théorique, CEA/Saclay, 91191 Gif-sur-Yvette Cedex, France

E-mail: mariana.grana@cea.fr, diego.marques@cea.fr

ABSTRACT: We find necessary and sufficient conditions for gauge invariance of the action of Double Field Theory (DFT) as well as closure of the algebra of gauge symmetries. The so-called weak and strong constraints are sufficient to satisfy them, but not necessary. We then analyze compactifications of DFT on twisted double tori satisfying the consistency conditions. The effective theory is a Gauged DFT where the gaugings come from the duality twists. The action, bracket, global symmetries, gauge symmetries and their closure are computed by twisting their analogs in the higher dimensional DFT. The non-Abelian heterotic string and lower dimensional gauged supergravities are particular examples of Gauged DFT.

KeYwords: Flux compactifications, String Duality

ARXIV EPRINT: 1201.2924 


\section{Contents}

1 Introduction 1

2 Double Field Theory 2

2.1 Gauge symmetries and constraints 3

3 Gauged Double Field Theory 5

3.1 The effective action $\quad 6$

$\begin{array}{lll}3.2 & \text { Gauge symmetries and constraints } & 7\end{array}$

3.3 Solutions to the constraints 9

3.4 Completing the action 11

4 Heterotic string as a GDFT 12

5 Conclusions $\quad 14$

\section{Introduction}

Double Field Theory (DFT) is a recent development that promotes a string duality to a symmetry in field theory. It is manifestly invariant under global symmetries that include T-duality transformations. The original formulation was done in [1-4], following previous ideas by Siegel $[5,6]$ and Tseytlin $[7,8]$ (see also $[9,10]$ ). Since then, DFT has been extended in many different ways [11-27]-[32]. Some very recent related works on duality based constructions are [33-40]. Some reviews on the subject can be found in [41, 42].

The theory is formally defined on a double space, that includes coordinates which are Fourier dual to momentum modes, plus T-dual coordinates associated to winding. However, the current formulation of DFT is restricted by the so-called weak and strong constraints, that ensure gauge invariance and closure of the algebra. Combined, these constraints imply that the theory is only consistent on a slice of the double space parameterized by half of the coordinates, such that there always exists a frame in which locally the configurations do not depend on dual coordinates. Therefore, the doubling is only formal and the theory is not truly doubled. Since the constraints are covariant under the global symmetries, the theory can still be covariantly formulated.

Recently, flux compactifications of DFT were considered in [30, 31]. It was realized that Scherk-Schwarz dimensional reductions [43] of DFT lead to gauged supergravities in lower dimensions, in which the so-called non-geometric fluxes [44-46] are purely geometric when analyzed from the point of view of the double space [47-51]. Interestingly, when the constraints are applied to the compactification ansatz, the gaugings in the effective theory are forced to satisfy relations that are stronger than the usual quadratic constraints 
(Jacobi identities) required for gauge consistency. The fact that the effective action is overconstrained, suggests that the constraints could be relaxed on the internal space.

In this paper, we explicitly show that it is possible to relax the weak and strong constraints. We first show that demanding gauge invariance and closure of the gauge transformations leads to a set of invariance and closure constraints. These constraints select subsets of fields and gauge parameters for which the gauge symmetries of DFT are consistent. Interestingly, the weak and strong constraints are sufficient to satisfy the closure and invariance constraints, but not necessary.

In [29] it was shown that DFT can be deformed by gaugings that preserve the global covariance of the theory. The gaugings allow for non-Abelian gauge symmetries for vectors, and therefore this scenario is fruitful for embeddings of the Heterotic String [29] and gauged supergravities $[30,31]$ in DFT. Here we show that these deformed theories, that we call Gauged DFT (GDFT), can be obtained from Scherk-Schwarz flux compactifications of higher dimensional (ungauged) DFTs. In this sense, the gaugings are not introduced by hand, but arise from the duality twists of the compactification and therefore have a higher dimensional origin. We show that the action of GDFT, its global symmetries, bracket, gauge symmetries and their closure, and the constraints can all be obtained by twisting their analogs in the parent higher dimensional DFT. The twisted constraints are such that their dependence on the gaugings always appears in the form of Jacobi-like quadratic constraints, plus terms that vanish if the strong and weak constraints are imposed on the fields and gauge parameters of the effective lower dimensional theory. Since the quadratic constraints for the gaugings are less restrictive than the weak and strong constraints, this implies that they can be relaxed on the internal space (a similar situation occurs for the strong constraint in the Ramond-Ramond sector of (massive) Type II theories [28]).

Finally, we show how the non-Abelian heterotic supergravity can be embedded in GDFT, by adding extra internal coordinates associated to the generators of the heterotic gauge symmetry, and twisting them to recover the structure constants of the nonAbelian algebra. Such a procedure is possible only after relaxing the constraints on the internal directions.

The paper is organized as follows. In section 2 we review the generalized metric formulation of DFT. We obtain the closure and invariance constraints required for consistency of gauge symmetries. Section 3 is devoted to analyze generalities of ScherkSchwarz dimensional reductions of DFT. In section 4 we review how to embed the 10dimensional non-Abelian heterotic String into GDFT. In section 5 we summarize our main results and conclude.

\section{Double Field Theory}

DFT is a field theory invariant under a global symmetry group $G$ with a $K$-dimensional fundamental representation (with indices $M, N=1, \ldots, K$ ), and a symmetric metric $\eta_{M N}$. The coordinates $X^{M}$ form fundamental vectors of $G$. To describe the NS degrees of freedom of string theory, one takes the group $G$ to be $O(K / 2, K / 2)$, but until section 4 we do not need to specify what $G$ is, we just need that it preserves a symmetric metric. 
The dynamical fields are the symmetric generalized metric $\mathcal{H}_{M N} \in G$, with inverse given by $\mathcal{H}^{M N}=\eta^{M P} \mathcal{H}_{P Q} \eta^{Q N}$, and a $G$ scalar $d$. The generalized metric can be written in terms of a generalized $K$-bein $\mathcal{E}^{a}{ }_{M}$

$$
\mathcal{H}_{M N}=\mathcal{E}^{a}{ }_{M} S_{a b} \mathcal{E}^{b}{ }_{N}
$$

where $S_{a b}$ is the planar metric in $K$ dimensions (where $a, b=1, \ldots, K$ ) with appropriate signature (i.e., for the case $G=O(D, D+d), S_{a b}=\operatorname{diag}(-1,1 \ldots 1 ;-1,1 \ldots 1 ; 1 \ldots 1)$ ).

The $G$ transformations act as

$$
X^{\prime M}=U^{M}{ }_{P} X^{P}, \quad \mathcal{E}^{\prime a}{ }_{M}\left(X^{\prime}\right)=\left(U^{-1}\right)^{P}{ }_{M} \mathcal{E}^{a}{ }_{P}(X), \quad d^{\prime}\left(X^{\prime}\right)=d(X), \quad U \in G .
$$

The dynamics of DFT is described by an action that can be written in a compact form (up to total derivatives) in terms of a generalized Ricci scalar as

$$
S_{D F T}=\int d^{K} X e^{-2 d} \mathcal{R}(\mathcal{E}, d)
$$

where $\mathcal{R}$ is defined by

$$
\begin{aligned}
\mathcal{R}(\mathcal{E}, d)= & 4 \mathcal{H}^{M N} \partial_{M} \partial_{N} d-\partial_{M} \partial_{N} \mathcal{H}^{M N}-4 \mathcal{H}^{M N} \partial_{M} d \partial_{N} d+4 \partial_{M} \mathcal{H}^{M N} \partial_{N} d \\
& +\frac{1}{8} \mathcal{H}^{M N} \partial_{M} \mathcal{H}^{K L} \partial_{N} \mathcal{H}_{K L}-\frac{1}{2} \mathcal{H}^{M N} \partial_{M} \mathcal{H}^{K L} \partial_{K} \mathcal{H}_{N L} \\
& +\frac{1}{2} \partial_{M} \mathcal{E}^{a}{ }_{P} \partial^{M} \mathcal{E}^{b}{ }_{Q} S_{a b} \eta^{P Q}
\end{aligned}
$$

Originally the coefficients in the first two lines were chosen by hand in order to require invariance of the action under the symmetries, but it was shown that this action (with the last term included) is actually up to total derivatives the Ricci scalar corresponding to the generalized metric, or to be more precise to a torsion free generalized connection $[5,6,32$, 33]. The last term vanishes if we impose the strong constraint, given below in (2.16), and is not in the original generalized metric formulation of DFT but we include it to recover the results of [33] and, as we will show in the next section, because it provides the right contribution to the dimensionally reduced theory.

\subsection{Gauge symmetries and constraints}

The infinitesimal gauge transformations are generated by parameters $\xi^{M}(X)$ in the fundamental representation of $G$, and take the form

$$
\begin{aligned}
\delta_{\xi} d & =\xi^{M} \partial_{M} d-\frac{1}{2} \partial_{M} \xi^{M} \\
\delta_{\xi} \mathcal{E}^{a}{ }_{M} & =\xi^{P} \partial_{P} \mathcal{E}^{a}{ }_{M}+\left(\partial_{M} \xi^{P}-\partial^{P} \xi_{M}\right) \mathcal{E}^{a}{ }_{P} .
\end{aligned}
$$

In general, one can define a gauge transformation for a generic tensor

$$
\delta_{\xi} V^{M}=\xi^{P} \partial_{P} V^{M}{ }_{N}+\left(\partial^{M} \xi_{P}-\partial_{P} \xi^{M}\right) V^{P}{ }_{N}+\left(\partial_{N} \xi^{P}-\partial^{P} \xi_{N}\right) V_{P}^{M}
$$


Here we illustrate the transformations using a tensor with an upper and a lower index such that the generalization to an arbitrary tensor is easy to obtain. These transformations define the so-called C-bracket

$$
\left[\xi_{1}, \xi_{2}\right]_{\mathrm{C}}^{M}=2 \xi_{[1}^{N} \partial_{N} \xi_{2]}^{M}-\xi_{[1}^{P} \partial^{M} \xi_{2] P}
$$

Demanding that the commutator of two transformations of an arbitrary given tensor $V^{M}{ }_{N}$ behaves as a transformation itself

$$
\left[\delta_{\xi_{1}}, \delta_{\xi_{2}}\right] V_{N}^{M}=\delta_{\left[\xi_{1}, \xi_{2}\right]_{\mathrm{C}}} V^{M}{ }_{N}-F^{M}{ }_{N}\left(\xi_{1}, \xi_{2}, V\right)
$$

leads to the following first closure constraint ${ }^{1}$

$$
F_{N}^{M}\left(\xi_{1}, \xi_{2}, V\right)=\xi_{[1}^{Q} \partial^{P} \xi_{2] Q} \partial_{P} V^{M}{ }_{N}+2 \partial_{P} \xi_{[1}^{Q} \partial^{P} \xi_{2] N} V^{M}{ }_{Q}+2 \partial_{P} \xi_{[1 Q} \partial^{P} \xi_{2]}^{M} V^{Q}{ }_{N}=0
$$

When fields and gauge parameters are restricted in such a way that one can always find a frame in which the dependence on dual coordinates vanishes, this equation is automatically satisfied. However, different configurations could be considered for which this is not the case.

The C-bracket has a non-vanishing Jacobiator, which can be written as

$$
\mathcal{J}_{\mathrm{C}}^{M}\left(\xi_{1}, \xi_{2}, \xi_{3}\right)=3\left[\xi_{[1},\left[\xi_{2}, \xi_{3]}\right]_{\mathrm{C}}\right]_{\mathrm{C}}^{M}=\frac{3}{2} \partial^{M}\left(\xi_{[1}^{P} \xi_{2}^{Q} \partial_{P} \xi_{3] Q}\right)+F^{M}\left(\xi_{[1}, \xi_{2}, \xi_{3]}\right)
$$

The last term of this equations vanishes due to the constraint (2.9). Imposing this constraint and using (2.8) the follow identity can be derived

$$
3\left[\delta_{\xi_{[1}},\left[\delta_{\xi_{2}}, \delta_{\left.\xi_{3}\right]}\right]\right]=\delta_{\mathcal{J}_{\mathrm{C}}\left(\xi_{1}, \xi_{2}, \xi_{3}\right)}
$$

Since the left hand side of (2.11) vanishes because the ordinary commutator satisfies the Jacobi identity, consistency requires that the Jacobiator generates trivial gauge transformations, and this leads to the second closure constraint

$$
H_{N}^{M}\left(\xi_{1}, \xi_{2}, \xi_{3}, V\right)=\delta_{\mathcal{J}_{\mathrm{C}}\left(\xi_{1}, \xi_{2}, \xi_{3}\right)} V^{M}{ }_{N}=\frac{3}{2} \partial^{R}\left(\xi_{[1}^{P} \xi_{2}^{Q} \partial_{P} \xi_{3] Q}\right) \partial_{R} V^{M}=0
$$

Again, for restricted configurations this is automatically satisfied, but it could admit other solutions.

The gauge transformations (2.5) imply that $\mathcal{R}$ and $e^{-2 d}$ transform as

$$
\delta_{\xi} \mathcal{R}=\xi^{M} \partial_{M} \mathcal{R}+G(\xi, \mathcal{E}, d), \quad \delta_{\xi} e^{-2 d}=\partial_{M}\left(\xi^{M} e^{-2 d}\right)
$$

\footnotetext{
${ }^{1}$ The index structure of the constraints (2.9) and (2.12) are those of the tensor acted upon by the commutator and Jacobiator.
} 
with

$$
\begin{aligned}
G(\xi, \mathcal{E}, d)= & -\partial^{P} \partial_{M} \xi_{N} \partial_{P} \mathcal{H}^{M N}-2 \partial^{P} \xi_{N} \partial_{P} \partial_{M} \mathcal{H}^{M N}+4 \partial_{P} d \partial_{M} \partial^{P} \xi_{N} \mathcal{H}^{M N} \\
& +4 \partial_{P} d \partial^{P} \xi_{N} \partial_{M} \mathcal{H}^{M N}+4 \partial_{N} d \partial^{P} \xi_{M} \partial_{P} \mathcal{H}^{M N} \\
& +\frac{1}{4} \mathcal{H}^{M N} \partial^{P} \xi_{M} \partial_{P} \mathcal{H}^{K L} \partial_{N} \mathcal{H}_{K L}-\mathcal{H}^{M N} \partial^{P} \xi_{M} \partial_{P} \mathcal{H}^{K L} \partial_{K} \mathcal{H}_{N L} \\
& +8 \mathcal{H}^{M N} \partial^{P} \xi_{M} \partial_{P} \partial_{N} d-8 \mathcal{H}^{M N} \partial_{M} d \partial^{P} \xi_{N} \partial_{P} \quad d \\
& -2 \partial_{M}\left(\partial^{P} \partial_{P} \xi_{N} \mathcal{H}^{M N}\right)+4 \partial^{P} \partial_{P} \xi_{M} \partial_{N} d \mathcal{H}^{M N} \\
& +\partial_{P} \xi^{Q} \partial_{Q} \mathcal{E}^{a}{ }_{M} \partial^{P} \mathcal{E}^{b}{ }_{N} S_{a b} \eta^{M N}+\partial_{P} \partial^{N} \xi^{M} \mathcal{E}^{a}{ }_{M} \partial^{P} \mathcal{E}^{b}{ }_{N} S_{a b} \\
& -\partial_{P} \partial^{M} \xi^{N} \mathcal{E}^{a}{ }_{M} \partial^{P} \mathcal{E}^{b}{ }_{N} S_{a b} .
\end{aligned}
$$

Therefore, for $\mathcal{R}$ to transform as a scalar, the following invariance constraint must hold

$$
\int d^{K} X e^{-2 d} G(\xi, \mathcal{E}, d)=0 .
$$

Also, for consistency, any subset of fields and gauge parameters allowed by the constraints must be such that under gauge transformations the transformed fields satisfy the constraints (2.9), (2.12) and (2.15) as well (i.e. the transformed fields must be also contained in the subset).

To summarize, consistency of gauge invariance in DFT requires two closure constraints (2.9) and (2.12) and the invariance constraint (2.15). All these restrictions involve contractions of two derivatives, and therefore vanish when the so-called weak and strong constraints hold, respectively

$$
\partial^{P} \partial_{P} V^{M}{ }_{N}=0, \quad \partial^{P} W^{R}{ }_{S} \partial_{P} V^{M}{ }_{N}=0,
$$

where $W$ and $V$ denote any field or gauge parameter. Imposing these constraints was shown to be extremely restrictive. In particular, they only allow for restricted configurations of fields and gauge parameters that can be locally rotated to a frame in which they do not depend on the dual coordinates. Here we see that these constraints are sufficient to satisfy the closure and invariance conditions, but not necessary.

\section{Gauged Double Field Theory}

In this section we compactify a $K$-dimensional DFT on a twisted torus of dimension $d$. This leads to an effective theory defined on an $N$-dimensional space with $N=K-d$, which we call GDFT. The resulting effective action depends on a set of $N$ external coordinates denoted $\mathbb{X}$, while the $d$ compact dimensions are referred to as $\mathbb{Y}$. The compactification is defined by the (duality) twist matrix

$$
U^{A}{ }_{M}(\mathbb{Y}) \in G,
$$


that maps the $G$ indices $M, N$ of the parent DFT into the $G$ indices $A, B$ of the effective GDFT. One starts by proposing a reduction ansatz that specifies the internal and external dependence of any arbitrary tensor

$$
V_{N}^{M}(\mathbb{X}, \mathbb{Y})=\left(U^{-1}\right)^{M}{ }_{A}(\mathbb{Y}) \widehat{V}^{A}{ }_{B}(\mathbb{X}) U^{B}{ }_{N}(\mathbb{Y}),
$$

where $V$ can denote either a field or a gauge parameter. The notation is such that the hatted tensors $\widehat{V}_{B}^{A}$ only depend on the coordinates $\mathbb{X}$, and correspond respectively to dynamical objects or gauge parameters in the effective action. The internal dependence is fixed by the twist matrix (3.1), and has no dynamics.

It must then be demanded that the $\mathbb{Y}$-dependence factorizes out of the gauge transformations and the action. The information on the internal space does not disappear however, and is encrypted in gaugings, which are $\mathbb{Y}$-independent combinations of derivatives of the twists (3.1).

At this point there are two conditions that can be imposed on the ansatz (3.2). One is that Lorentz invariance of the $N$-dimensional effective action is preserved, which requires that Lorentzian coordinates remain untwisted, so any $\mathbb{X}$-dependent quantity $\widehat{g}(\mathbb{X})$ must satisfy

$$
\left(U^{-1}\right)^{M}{ }_{A} \partial_{M} \widehat{g}(\mathbb{X})=\partial_{A} \widehat{g}(\mathbb{X}) .
$$

Also, we restrict the set of external and internal coordinates in such a way that if a given coordinate is external (internal), its dual must be external (internal). This can be imposed through

$$
\partial^{P} U^{A}{ }_{M} \partial_{P} \widehat{g}(\mathbb{X})=0,
$$

and it ensures that the effective GDFT is also formally doubled.

\subsection{The effective action}

To obtain the effective action of GDFT, we start by twisting the generalized metric, and provide a scalar twist $\lambda(\mathbb{Y})$ to the $d$ field

$$
\mathcal{E}^{a}{ }_{M}(\mathbb{X}, \mathbb{Y})=U^{B}{ }_{M}(\mathbb{Y}) \widehat{\mathcal{E}}^{a}{ }_{B}(\mathbb{X}), \quad d(\mathbb{X}, \mathbb{Y})=\widehat{d}(\mathbb{X})+\lambda(\mathbb{Y})
$$

When (3.5) is inserted in (2.3) the action of GDFT is obtained

$$
S_{G D F T}=v \int d^{N} \mathbb{X} e^{-2 \widehat{d}}\left(\mathcal{R}(\widehat{\mathcal{E}}, \widehat{d})+\mathcal{R}_{f}(\widehat{\mathcal{E}}, \widehat{d})\right),
$$

where $v$ is defined by

$$
v=\int d^{d} \mathbb{Y} e^{-2 \lambda(\mathbb{Y})},
$$

and $\mathcal{R}_{f}$ is the gauged part of the action

$$
\begin{aligned}
\mathcal{R}_{f}= & -\frac{1}{2} f^{A}{ }_{B C} \widehat{\mathcal{H}}^{B D} \widehat{\mathcal{H}}^{C E} \partial_{D} \widehat{\mathcal{H}}_{A E}-\frac{1}{12} f^{A}{ }_{B C} f^{D}{ }_{E F} \widehat{\mathcal{H}}_{A D} \widehat{\mathcal{H}}^{B E} \widehat{\mathcal{H}}^{C F} \\
& -\frac{1}{4} f^{A}{ }_{B C} f^{B}{ }_{A D} \widehat{\mathcal{H}}^{C D}-2 f_{A} \partial_{B} \widehat{\mathcal{H}}^{A B}+4 f_{A} \widehat{\mathcal{H}}^{A B} \partial_{B} \widehat{d}-f_{A} f_{B} \widehat{\mathcal{H}}^{A B},
\end{aligned}
$$


where

$$
\widehat{\mathcal{H}}_{A B}(\mathbb{X})=\widehat{\mathcal{E}}^{a}{ }_{A}(\mathbb{X}) S_{a b} \widehat{\mathcal{E}}^{b}{ }_{B}(\mathbb{X}) .
$$

All the dependence on the twists enters in the effective action through the overall scale $v$ in (3.7) and the gaugings

$$
\begin{aligned}
f_{A B C} & =3 \eta_{D[A}\left(U^{-1}\right)^{M}{ }_{B}\left(U^{-1}\right)^{N}{ }_{C]} \partial_{M} U^{D}{ }_{N}, \\
f_{A} & =\partial_{M}\left(U^{-1}\right)^{M}{ }_{A}-2\left(U^{-1}\right)^{M}{ }_{A} \partial_{M} \lambda,
\end{aligned}
$$

which are taken to be constant. Note that the restriction (3.3) implies the following consistency constraint

$$
f^{A}{ }_{B C} \partial_{A} \widehat{g}(\mathbb{X})=0, \quad f^{A} \partial_{A} \widehat{g}(\mathbb{X})=0 .
$$

This states that the $N$-dimensional Lorentz invariance is not broken in the effective action by the gaugings.

If we had not included the last term in the definition of the generalized Ricci scalar (2.4), this action would involve an additional term proportional to the twists $U$, which are $\mathbb{Y}$-dependent. Since we have demanded all the $\mathbb{Y}$-dependence to factorize out, we are forced to include such additional term.

\subsection{Gauge symmetries and constraints}

The gauge parameters $\widehat{\xi}^{A}$ in GDFT are obtained by twisting the gauge parameter $\xi^{M}$ of the parent DFT

$$
\xi^{M}(\mathbb{X}, \mathbb{Y})=\left(U^{-1}\right)^{M}(\mathbb{Y}) \widehat{\xi}^{A}(\mathbb{X})
$$

The effective gauge transformations $\widehat{\delta}_{\widehat{\xi}}$ are defined through the relation

$$
\delta_{\xi} V^{M}=\left(U^{-1}\right)^{M}{ }_{A} U^{B}{ }_{N} \widehat{\delta}_{\widehat{\xi}} \widehat{V}_{B}^{A},
$$

which guarantees that the transformed tensors maintain the "Scherk-Schwarz" structure (3.2). After some algebra the following gauge transformation can be extracted from $(3.13)$

$$
\begin{aligned}
\widehat{\delta}_{\widehat{\xi}} \widehat{V}^{A}{ }_{B}= & \widehat{\xi}^{C} \partial_{C} \widehat{V}_{B}^{A}+\left(\partial^{A} \widehat{\xi}_{C}-\partial_{C} \widehat{\xi}^{A}\right) \widehat{V}^{C}{ }_{B}+\left(\partial_{B} \widehat{\xi}^{C}-\partial^{C} \widehat{\xi}_{B}\right) \widehat{V}^{A}{ }_{C} \\
& -f^{A}{ }_{C D} \widehat{\xi}^{C} \widehat{V}^{D}{ }_{B}+f^{D}{ }_{C B} \widehat{\xi}^{C} \widehat{V}^{A}{ }_{D} .
\end{aligned}
$$

Note that the first line is the usual gauge transformation of the ungauged theory. Again, for consistency the gaugings must be constant to ensure that gauge transformations do not reintroduce $\mathbb{Y}$-dependence. For the fields of GDFT the gauge transformations (3.14) read

$$
\widehat{\delta}_{\vec{\xi}^{-\mathcal{E}^{a}}}{ }_{B}=\widehat{\xi}^{C} \partial_{C} \widehat{\mathcal{E}}^{a}{ }_{B}+\left(\partial_{B} \widehat{\xi}^{C}-\partial^{C} \widehat{\xi}_{B}\right) \widehat{\mathcal{E}}^{a}{ }_{C}+f^{D}{ }_{C B} \widehat{\xi}^{C} \widehat{\mathcal{E}}^{a}{ }_{D}
$$

and

$$
\begin{aligned}
& \widehat{\delta}_{\vec{\xi}} \widehat{d}(\mathbb{X})=\widehat{\xi}^{A} \partial_{A} \widehat{d}-\frac{1}{2} \partial_{A} \widehat{\xi}^{A}-\frac{1}{2} f_{A} \widehat{\xi}^{A} \\
& \widehat{\delta}_{\widehat{\xi}} e^{-2 \widehat{d}}=\delta_{\xi} e^{-2 d}=\partial_{A}\left(\widehat{\xi}^{A} e^{-2 \widehat{d}}\right)+f_{A} \widehat{\xi}^{A} e^{-2 \widehat{d}}
\end{aligned}
$$


The C-bracket defines a twisted effective bracket, which we call $f$-bracket $[,]_{f}$, through the relation

$$
\left[\xi_{1}, \xi_{2}\right]_{\mathrm{C}}^{M}=\left(U^{-1}\right)^{M}{ }_{A}\left[\widehat{\xi}_{1}, \widehat{\xi}_{2}\right]_{f}^{A} .
$$

The $f$-bracket preserves the structure of the C-Bracket but receives an extra contribution from the gaugings

$$
\left[\widehat{\xi}_{1}, \widehat{\xi}_{2}\right]_{f}^{A}=\left[\widehat{\xi}_{1}, \widehat{\xi}_{2}\right]_{\mathrm{C}}^{A}-f^{A}{ }_{B D} \widehat{\xi}_{1}^{B} \widehat{\xi}_{2}^{D} .
$$

Closure of the effective gauge transformations also gives rise to the $f$-bracket plus twisted closure constraints (cf. equation (2.8))

$$
\left[\widehat{\delta}_{\widehat{\xi}_{1}}, \widehat{\delta}_{\widehat{\xi}_{2}}\right] \widehat{V}_{B}^{A}=\widehat{\delta}_{\left[\widehat{\xi}_{1}, \widehat{\xi}_{2}\right]_{f}} \widehat{V}_{B}^{A}-\widehat{F}_{B}^{A}\left(\widehat{\xi}_{1}, \widehat{\xi}_{2}, \widehat{V}\right)
$$

Using that the gaugings are constant, we get after some algebra the first twisted closure constraint

$$
\begin{aligned}
\widehat{F}^{A}{ }_{B}\left(\widehat{\xi}_{1}, \widehat{\xi}_{2}, \widehat{V}\right)= & U^{A}{ }_{M}\left(U^{-1}\right)^{N}{ }_{B} F^{M}{ }_{N}\left(\xi_{1}, \xi_{2}, V\right) \\
= & \widehat{\xi}_{[1}^{C} \partial^{D} \widehat{\xi}_{2] C} \partial_{D} \widehat{V}_{B}^{A}+2 \partial_{D} \widehat{\xi}_{[1}^{C} \partial^{D} \widehat{\xi}_{2] B} \widehat{V}^{A}{ }_{C}+2 \partial_{D} \widehat{\xi}_{[1 C} \partial^{D} \widehat{\xi}_{2]}^{A} \widehat{V}_{B}^{C} \\
& \left.\quad-3 f_{F[C D} f_{E]}^{F}{ }_{B} \widehat{\xi}_{[1}^{C} \widehat{\xi}_{2]}^{D} \widehat{V}^{E}{ }_{B}-3 f^{F[C D} f_{F} E\right]{ }_{B} \widehat{\xi}_{[1 C} \widehat{\xi}_{2] D} \widehat{V}_{E}^{A}=0
\end{aligned}
$$

which can be obtained either by twisting the first closure constraint in the higher dimensional DFT, or by demanding that two transformations (3.14) reproduce a unique transformation. The second line in $(3.20)$ is $F\left(\widehat{\xi}_{1}, \widehat{\xi}_{2}, \widehat{V}\right)$, and the last line is generated by gaugings that interestingly are arranged in a Jacobi-like form.

The Jacobiator $\widehat{\mathcal{J}}_{f}$ of the $f$-bracket is given again by a total derivative

$$
\widehat{\mathcal{J}}_{f}\left(\widehat{\xi}_{1}, \widehat{\xi}_{2}, \widehat{\xi}_{3}\right)^{D}=\frac{3}{2} \partial^{D}\left(\widehat{\xi}_{[1}^{A} \widehat{\xi}_{2}^{B} \partial_{A} \widehat{\xi}_{3] B}+\frac{1}{3} f_{A B C} \widehat{\xi}_{1}^{A} \widehat{\xi}_{2}^{B} \widehat{\xi}_{3}^{C}\right)
$$

and demanding that it generates trivial gauge transformations leads to the second twisted closure constraint

$$
\begin{aligned}
\widehat{H}_{F}^{E}\left(\widehat{\xi}_{1}, \widehat{\xi}_{2}, \widehat{\xi}_{3}, \widehat{V}\right) & =U^{E}{ }_{M}\left(U^{-1}\right)^{N}{ }_{F} H^{M}{ }_{N}\left(\xi_{1}, \xi_{2}, \xi_{3}, V\right) \\
& =\frac{3}{2} \partial^{D}\left(\widehat{\xi}_{[1}^{A} \widehat{\xi}_{2}^{B} \partial_{A} \widehat{\xi}_{3] B}+\frac{1}{3} f_{A B C} \widehat{\xi}_{1}^{A} \widehat{\xi}_{2}^{B} \widehat{\xi}_{3}^{C}\right) \partial_{D} \widehat{V}_{F}^{E}=0 .
\end{aligned}
$$

Again this expressions can be found either by twisting their analogs in the higher dimensional DFT, or by direct computations using the $f$-bracket and the constraint (3.20).

The deformed Ricci scalar (3.6) can be shown to transform as follows under gauge transformations (3.15) and (3.16)

$$
\widehat{\delta}_{\widehat{\xi}}\left(\mathcal{R}(\widehat{\mathcal{E}}, \widehat{d})+\mathcal{R}_{f}(\widehat{\mathcal{E}}, \widehat{d})\right)=\widehat{\xi}^{A} \partial_{A}\left(\mathcal{R}(\widehat{\mathcal{E}}, \widehat{d})+\mathcal{R}_{f}(\widehat{\mathcal{E}}, \widehat{d})\right)+G(\widehat{\xi}, \widehat{\mathcal{E}}, \widehat{d})+G_{f}(\widehat{\xi}, \widehat{\mathcal{E}}, \widehat{d}),
$$

where

$$
\begin{aligned}
G_{f}(\widehat{\xi}, \widehat{\mathcal{E}}, \widehat{d})= & G(\xi, \mathcal{E}, d)-G(\widehat{\xi}, \widehat{\mathcal{E}}, \widehat{d}) \\
= & \frac{1}{2} \widehat{\mathcal{H}}^{A B} \partial^{D} \widehat{\xi}_{A} \partial_{D} \widehat{\mathcal{H}}^{E F} \widehat{\mathcal{H}}_{G F} f_{B E}^{G}+\frac{1}{2} \widehat{\xi}_{C} \widehat{\mathcal{H}}^{A B} \widehat{\mathcal{H}}^{E F} \widehat{\mathcal{H}}^{G H} f_{D[A}{ }^{C} f^{D}{ }_{G E]} f_{B F H} \\
& -\frac{3}{2} \widehat{\xi}^{C} \widehat{\mathcal{H}}^{A B} \widehat{\mathcal{H}}^{E F} \partial_{B} \widehat{\mathcal{H}}_{G F} f_{D[A C} f^{D}{ }_{E]}{ }^{G}-\frac{3}{2} \widehat{\xi}^{G} \widehat{\mathcal{H}}^{D H} f^{A C}{ }_{[B} f_{G H] C} f^{B}{ }_{A D} \\
& -f_{A}{ }^{B C} \widehat{\mathcal{E}}^{a}{ }_{B} \partial_{D} \widehat{\mathcal{E}}^{b}{ }_{C} S_{a b} \partial^{D} \widehat{\xi}^{A}
\end{aligned}
$$


provided the gaugings $f_{A}$ vanish. Let us briefly explain in the context of the higher dimensional theory why this should be the case. The parent DFT transforms as a total derivative involving the duality twists, which are not globally well defined. Therefore, to ensure gauge invariance, one should impose as a constraint for the duality twists that such derivative vanishes. Since it is proportional to $f_{A}$, these gaugings must be set to zero to ensure gauge invariance in the effective theory. In [31] it was shown how to introduce $f_{A}$ deformations through modifications of the ansatz that involve a warp factor. We will not consider this possibility here. Due to (3.23) the action (2.3) is then gauge invariant when the following twisted invariance constraints hold

$$
v \int d^{N} \mathbb{X} e^{-2 \widehat{d}}\left[G(\widehat{\xi}, \widehat{\mathcal{E}}, \widehat{d})+G_{f}(\widehat{\xi}, \widehat{\mathcal{E}}, \widehat{d})\right]=0, \quad f_{A}=0
$$

The action of GDFT formally preserves the $G$ covariance of the original theory. However, the gaugings explicitly break this group and gauge a subgroup of it. It they were treated as spurions, and rotated under $G$, both the action and gauge transformations would transform covariantly. This is in complete analogy with gauged supergravities [52]. For a given gauging, the effective theory contains vectors preserving a non-Abelian gauge symmetry, and this is the reason why we call this theory Gauged DFT. A concrete realization of this fact is given in section 4, where we analyze the embedding of the non-Abelian heterotic supergravity in GDFT.

\subsection{Solutions to the constraints}

In this section we present a possible family of solutions to the effective constraints (3.20), (3.22) and (3.25). We require the effective tensors $\widehat{V}$ and $\widehat{W}$ to satisfy the weak and strong constraints on the external space

$$
\partial_{E} \partial^{E} \widehat{V}_{B}^{A}=0, \quad \partial_{E} \widehat{V}_{B}^{A} \partial^{E} \widehat{W}^{C}{ }_{D}=0 .
$$

In this case, (3.20) dictates that the gaugings should satisfy Jacobi identities

$$
f_{E[A B} f_{C] D}{ }^{E}=0 .
$$

The other constraints, equations (3.22) and (3.25) are then automatically satisfied. We now argue that in general these configurations do not satisfy the strong and weak constraints (2.16).

It is convenient to define the tensor

$$
\Omega_{A B C}=\eta_{C D}\left(U^{-1}\right)^{M}{ }_{A}\left(U^{-1}\right)^{N}{ }_{B} \partial_{M} U^{D}{ }_{N}=-\Omega_{A C B},
$$

related to the gaugings through

$$
f_{A B C}=3 \Omega_{[A B C]} .
$$

In terms of this object, the strong constraint for the duality twists

$$
\partial_{P} U^{A}{ }_{M} \partial^{P} U^{B}{ }_{N}=0,
$$


implies

$$
\Omega_{E A B} \Omega^{E}{ }_{C D}=0
$$

while Jacobi identities read [31]

$$
f_{E[A B} f_{C] D}^{E}=\Omega_{E[A B} \Omega^{E}{ }_{C] D}-4 U^{E}{ }_{M} \eta_{E[A} \partial^{M} f_{B C D]}=0 .
$$

Since the gaugings are constant, the last term in (3.32) vanishes. Given that (3.31) appears antisymmetrized in (3.32), the strong constraint suffices to satisfy all the effective constraints, but it is not necessary.

This observation was also made in [31], and the fact that the strong constraint is never used in the compactification procedure was previously noted in [30]. In the light of our results, we now see that relaxing the strong constraint on the internal space is a perfectly consistent choice.

As for the weak constraint, we note that if we require the twist to satisfy it, given that $U \in G$, we get that the following condition holds

$$
\Omega^{C D A} \Omega_{C D}{ }^{B}=0,
$$

which is not required by our constraint (3.27). So similarly to the strong constraint, we get that the weak constraint is more restrictive than what we need for invariance of the action and closure of the algebra.

We would also like to point out the difficulty in relaxing the strong constraint while keeping the weak. In fact, for a set of fields and gauge parameters satisfying the weak constraint to be consistent, under gauge transformations the transformed fields must also satisfy this restriction. However, the transformed fields are given by products of (untransformed) fields and gauge parameters, so for the weak constraint to annihilate the transformed field, the untransformed ones must obey strong-like constraints. Let us also comment on what would happen if the weak constraint were imposed on the solutions (3.26)(3.27). Notice that if the generalized metric together with the gauge parameters were restricted to satisfy the weak constraint, one obtains using (3.4)

$$
\begin{aligned}
\partial_{M} \partial^{M} \xi_{P} & =\partial_{M} \partial^{M} U^{A}{ }_{P} \widehat{\xi}_{A}+U^{A}{ }_{P} \partial_{M} \partial^{M} \widehat{\xi}_{A}=0 \\
\partial_{M} \partial^{M} \mathcal{H}_{P Q} & =\partial_{M} \partial^{M} U^{A}{ }_{P} \widehat{\mathcal{H}}_{A B} U^{B}{ }_{Q}+U^{A}{ }_{P} \widehat{\mathcal{H}}_{A B} \partial_{M} \partial^{M} U^{B}{ }_{Q} \\
& +2 \partial_{M} U^{A}{ }_{P} \widehat{\mathcal{H}}_{A B} \partial^{M} U^{B}{ }_{Q}+U^{A}{ }_{P} \partial_{M} \partial^{M} \widehat{\mathcal{H}}_{A B} U^{B}{ }_{Q}=0
\end{aligned}
$$

The last terms in these equations vanish due to (3.26). Also, since the effective gauge parameters are allowed to take values in any direction, then (3.34) implies that the first two terms of (3.35) vanish also. Therefore, the third term in (3.35) must vanish

$$
\Omega_{E A(B} \Omega_{C) D}^{E}=0 .
$$

Since $\Omega^{E}{ }_{A B}=-\Omega^{E}{ }_{B A}$, we conclude that $\Omega_{E A B} \Omega^{E}{ }_{C D}=\Omega_{E[A B} \Omega^{E}{ }_{C D]}$, which vanishes due to the Jacobi identities and constancy of the gaugings (3.32). Therefore, when the weak constraint is combined with the Jacobi identities, the strong constraint (3.31) must hold for the duality twists in the solutions (3.26)-(3.27). 
Let us stress that the weak constraint is derived in string theory from a worldsheet analysis in the context of Kaluza-Klein compactifications of closed strings on tori. It is possible, in light of our results based on a field theory analysis, that such constraint could be modified in Scherk-Schwarz compactifications of closed strings on more general spaces like the ones we consider in this paper. These issues deserve a better understanding and we hope to return to them in the future.

Although the weak and strong constraints can be relaxed on the internal space, consistency requires other constraints which can also be highly restrictive (although, in principle allow for truly double spaces). As we said, the weak and strong constraints must be replaced by the Jacobi identities (or more generally, the twisted constraints). The condition that the gaugings must be constant restrict the possible configurations also. In addition, the duality twist is forced to be an element of $G$, and the condition $f_{A}=0$ obtained in (3.25) (where $f_{A}$ are defined in (3.10)) poses a condition on $\lambda(\mathbb{Y})$. Regarding global aspects, since the duality twist can be thought of as a generalized $K$-bein [51], the differentials $U^{A}{ }_{M} d \mathbb{Y}^{M}$ must be globally well defined up to identifications of coordinates. Finally, there are certain extra conditions on the algebra that have to be met such that one can consistently find a set of identifications to make the space compact (a discussion within solvable algebras can be found in [53] and references therein). We will not discuss them here. Interesting works on how to generate gaugings from duality twists are [51, 54].

\subsection{Completing the action}

As pointed out in [30], when the effective action (3.6) is written in the form of gauged halfmaximal supergravity [55], it differs from the latter by the following term in the Lagrangian

$$
-e^{-2 \widehat{d}} \frac{1}{6} f_{A B C} f^{A B C} \text {. }
$$

The absence of such term indicates that the theory actually corresponds to a truncation of maximal supergravity [56, 57]. Comparing our results with those of [29], we see that both actions differ by exactly the same term.

This term can have a higher dimensional origin in the following manifestly $G$-invariant additional piece in the action [31]

$$
\triangle S_{D F T}=-\frac{1}{6} \int d^{N} \mathbb{X} d^{d} \mathbb{Y} e^{-2 d} \mathcal{F}_{a b c} \mathcal{F}^{a b c}=\int d^{N} \mathbb{X} d^{d} \mathbb{Y} e^{-2 d} \triangle \mathcal{R},
$$

where the indices are contracted using the metric $\eta_{a b}$, which coincides with the metric of the group $G$. The tensor $\mathcal{F}_{a b c}$ is given by

$$
\mathcal{F}_{a b c}=3 S_{d[a}\left(\mathcal{E}^{-1}\right)^{M}{ }_{b}\left(\mathcal{E}^{-1}\right)^{N}{ }_{c]} \partial_{M} \mathcal{E}^{d}{ }_{N} .
$$

The consistency constraints (2.9), (2.12) and (3.25) do not require the term (3.38) to be zero. The overall factor $-\frac{1}{6}$ in $(3.38)$ is not fixed by the symmetries, but chosen by hand to reproduce the corresponding term in gauged half-maximal supergravities $[30,31]$ and the heterotic string [29]. 
Under the gauge transformations (2.5), this contribution to the generalized Ricci scalar transforms as

$$
\delta_{\xi}(\triangle \mathcal{R})=\xi^{M} \partial_{M}(\triangle \mathcal{R})+\triangle G(\xi, \mathcal{E})
$$

where

$$
\triangle G(\xi, \mathcal{E})=-\partial_{P} \xi^{Q} \partial_{Q} \mathcal{E}^{a}{ }_{M} \partial^{P} \mathcal{E}^{b}{ }_{N} \eta_{a b} \eta^{M N}+2 \partial_{P} \xi^{N} \partial^{M} \mathcal{E}^{a}{ }_{N} \partial^{P} \mathcal{E}^{b}{ }_{M} \eta_{a b},
$$

which contributes to the total $G$ in (2.14).

When evaluated in the ansatz (3.5), the tensor (3.39) splits into the contributions

$$
\mathcal{F}_{a b c}=\widehat{\mathcal{F}}_{a b c}+f_{D E F}\left(\widehat{\mathcal{E}}^{-1}\right)^{D}{ }_{a}\left(\widehat{\mathcal{E}}^{-1}\right)^{E}{ }_{b}\left(\widehat{\mathcal{E}}^{-1}\right)^{F}{ }_{c},
$$

where

$$
\widehat{\mathcal{F}}_{a b c}=3 S_{d[a}\left(\widehat{\mathcal{E}}^{-1}\right)^{E}{ }_{b}\left(\widehat{\mathcal{E}}^{-1}\right)^{F}{ }_{c]} \partial_{E} \widehat{\mathcal{E}}^{d}{ }_{F},
$$

and leads to two extra terms in the effective action

$$
\begin{aligned}
\triangle S_{G D F T} & =-\frac{1}{6} v \int d^{N} \mathbb{X} e^{-2 \widehat{d}}\left(f_{A B C} f^{A B C}+\widehat{\mathcal{F}}_{a b c} \widehat{\mathcal{F}}^{a b c}\right) \\
& =-\frac{1}{6} v \int d^{N} \mathbb{X} e^{-2 \widehat{d}}\left(\triangle R_{f}+\triangle R(\widehat{\mathcal{E}})\right) .
\end{aligned}
$$

Under the effective gauge transformations (3.15), the last term in (3.44) transforms as

$$
\widehat{\delta}_{\widehat{\xi}} \triangle R(\widehat{\mathcal{E}})=\widehat{\xi}^{C} \partial_{C} \triangle R(\widehat{\mathcal{E}})+\triangle G(\widehat{\xi}, \widehat{\mathcal{E}})+\triangle G_{f}(\widehat{\xi}, \widehat{\mathcal{E}})
$$

with

$$
\triangle G_{f}(\widehat{\xi}, \widehat{\mathcal{E}})=\triangle G(\xi, \mathcal{E})-\triangle G(\widehat{\xi}, \widehat{\mathcal{E}})=f_{A}^{B C} \widehat{\mathcal{E}}^{a}{ }_{B} \partial_{D} \widehat{\mathcal{E}}^{b}{ }_{C} \partial^{D} \widehat{\xi}^{A} \eta_{a b},
$$

contributing to $G_{f}$ in (3.24). The first term in equation (3.44) gives precisely the required contribution to complete the results of [29]. It can be checked that the second term in (3.44) vanishes using a standard parametrization (lower triangular for the case of $O(K / 2, K / 2)$ ) for the $K$-bein (see $[51,58]$ ) if the weak and strong constraints are satisfied on the external space. In this situation, whenever $\widehat{\mathcal{F}}_{a b c}$ in non-zero, $\widehat{\mathcal{F}}^{a b c}$ vanishes. To understand this intuitively, one can think of an analogous situation for the first term. This terms corresponds to contractions between geometric and non-geometric fluxes, and is zero in situations where the weak and strong constraints apply to the internal space and the twist is given a geometric parametrization. The setup in the next section is precisely the one under which the second term in (3.44) vanishes.

\section{Heterotic string as a GDFT}

In this section we would like to revisit the results of [29], and show how the non-Abelian heterotic strings $[59,60]$ can be embedded in GDFT (see [61] for a discussion of the Abelian case). The first re-writing of the heterotic in a $G$ covariant way was done in [62]. It is not surprising that both the heterotic supergravity and half-maximal gauged supergravity in four dimensions can be embedded in GDFT, since the later can be obtained from dimensional reductions of the former (see [63] and references therein). 
The novel feature here is that the structure constants of the non-Abelian gauge group are not put in by hand, but have now a higher dimensional origin in the twist of a $d$ dimensional space. The starting DFT has global symmetry group $G=O(D, D+d)$, with $K=D+D+d$ and metric

$$
\eta_{M N}=\left(\begin{array}{ccc}
0 & 1_{D} & 0 \\
1_{D} & 0 & 0 \\
0 & 0 & 1_{d}
\end{array}\right)
$$

where $1_{D}$ is the $D \times D$ identity matrix. The coordinates are fundamental vectors grouped according to

$$
X^{M}=\left(\tilde{x}_{i}, x^{i}, y^{\alpha}\right),
$$

with $i=1, \ldots, D, \alpha=1, \ldots, d$. The generalized $(2 D+d) \times(2 D+d)$ metric is twisted as in (3.5), where the "effective" generalized metric $\widehat{\mathcal{H}}$ is parameterized by the $D$-dimensional metric $g_{i j}$, the $D$-dimensional Kalb-Ramond form $B_{i j}$ and $d D$-dimensional vectors $A_{i}{ }^{\alpha}$

$$
\widehat{\mathcal{H}}_{M N}=\left(\begin{array}{ccc}
g^{i j} & -g^{i k} c_{k j} & -g^{i k} A_{k \beta} \\
-g^{j k} c_{k i} & g_{i j}+c_{k i} g^{k l} c_{l j}+A_{i}^{\gamma} A_{j \gamma} & c_{k i} g^{k l} A_{l \beta}+A_{i \beta} \\
-g^{j k} A_{k \alpha} & c_{k j} g^{k l} A_{l \alpha}+A_{j \alpha} & \delta_{\alpha \beta}+A_{k \alpha} g^{k l} A_{l \beta}
\end{array}\right),
$$

with

$$
c_{i j}=B_{i j}+\frac{1}{2} A_{i}{ }^{\alpha} A_{j \alpha} .
$$

The other generalized field is defined by the $G$ invariant combination of the dilaton $\phi$ and the determinant of the metric $g$

$$
e^{-2 \widehat{d}}=\sqrt{g} e^{-2 \phi} .
$$

We consider the particular case in which $N=2 D=10+10, d=496$, and define $\mathbb{X}=(\tilde{x}, x)$ and $\mathbb{Y}=y$. The $d$ internal coordinates $y^{\alpha}$ are twisted, and to recover the low energy action of the heterotic string we will require no dependence on the dual external coordinates $\tilde{x}_{i}$. The gaugings take the form

$$
f^{A}{ }_{B C}=\left\{\begin{array}{ll}
f^{\alpha}{ }_{\beta \gamma} & \text { if }(A, B, C)=(\alpha, \beta, \gamma) \\
0 & \text { otherwise }
\end{array},\right.
$$

and are constrained by

$$
f_{[\alpha \beta}^{\delta} f_{\gamma] \delta}^{\epsilon}=0
$$

In this case the constraints for external fields (3.20), (3.22) and (3.25) are trivially satisfied due to the fact that the fields do not depend on the coordinates $\tilde{x}$. Notice that for the twisted internal space the metric (4.1) is the identity. Therefore, if the weak and strong constraints acted in the twisted internal space, they would remove all the dependence on $y^{\alpha}$, and therefore the gaugings would vanish, leaving the effective theory with an Abelian gauge symmetry.

Under these assumptions and with these parameterizations, the action of GDFT (3.6) completed with the extra contribution (3.44) can be cast in the form

$$
S_{\mathrm{Het}}=v \int d^{10} x \sqrt{g} e^{-2 \phi}\left[R+4 \partial_{i} \phi \partial^{i} \phi-\frac{1}{12} \hat{H}_{i j k} \hat{H}^{i j k}-\frac{1}{4} \delta_{\alpha \beta} F_{i j}^{\alpha} F^{i j \beta}\right],
$$


where $R$ is the 10-dimensional Ricci scalar, and we have defined the curvatures

$$
\hat{H}_{i j k}=3\left(\partial_{[i} B_{j k]}-\delta_{\alpha \beta} A_{[i}{ }^{\alpha} \partial_{j} A_{k]}{ }^{\beta}\right)+\delta_{\alpha \sigma} f^{\sigma}{ }_{\beta \gamma} A_{[i}{ }^{\alpha} A_{j}{ }^{\beta} A_{k]}{ }^{\gamma},
$$

and

$$
F_{i j}^{\alpha}=2 \partial_{[i} A_{j]}^{\alpha}+f^{\alpha}{ }_{\beta \gamma} A_{[i}^{\beta} A_{j]}^{\gamma} .
$$

Regarding the gauge symmetries of this theory, they are inherited from the gauge symmetries of GDFT through (3.15). When evaluating the different components of (3.15) one finds the following transformation properties for the fields

$$
\begin{aligned}
\widehat{\delta}_{\widehat{\xi}} g^{i j} & =\varepsilon^{k} \partial_{k} g^{i j}-2 \partial_{k} \varepsilon^{(i} g^{j) k}, \\
\widehat{\delta}_{\widehat{\xi}} A_{i}^{\alpha} & =\varepsilon^{k} \partial_{k} A_{i}^{\alpha}+\partial_{i} \varepsilon^{k} A_{k}^{\alpha}+\partial_{i} \Lambda^{\alpha}-f^{\alpha}{ }_{\beta \gamma} \Lambda^{\beta} A_{i}{ }^{\gamma}, \\
\widehat{\delta}_{\widehat{\xi}} B_{i j} & =\varepsilon^{k} \partial_{k} B_{i j}+2 \partial_{[i} \varepsilon^{k} B_{j] k}+2 \partial_{[i} \tilde{\varepsilon}_{j]}+A_{[i}{ }^{\alpha} \partial_{j]} \Lambda_{\alpha}, \\
\widehat{\delta}_{\overparen{\xi}} \phi & =\varepsilon^{k} \partial_{k} \phi,
\end{aligned}
$$

where we have parameterized $\widehat{\xi}^{A}=\left(\tilde{\varepsilon}_{i}, \varepsilon^{i}, \Lambda^{\alpha}\right)$. This is precisely the way under which the fields transform under diffeomorphisms parameterized by $\varepsilon^{i}$, gauge transformations of the $B$-field parameterized by $\tilde{\varepsilon}_{i}^{\prime}=\tilde{\varepsilon}_{i}-\frac{1}{2} A_{i}{ }^{\alpha} \Lambda_{\alpha}$ and gauge transformations of the heterotic gauge fields parameterized by $\Lambda^{\alpha}[29]$.

The action (4.8) together with its transformation laws (4.11)-(4.14) are precisely those of the non-Abelian heterotic 10-dimensional supergravity, provided the structure constants $\omega^{a} b c$ of the $E_{8} \times E_{8}$ or $\mathrm{SO}(32)$ group of the Heterotic are identified with the gaugings $f^{\alpha}{ }_{\beta \gamma}$ in a basis in which the Killing form $\kappa_{a b}$ coincides with the metric $\delta_{\alpha \beta}$. To make the connection explicit, we define a change of basis $\nu$ and the heterotic gauge coupling $g_{0}$

$$
\kappa_{a b}=\nu_{a}^{\alpha} \delta_{\alpha \beta} \nu_{b}^{\beta}, \quad \delta_{\alpha \beta}=\nu^{a}{ }_{\alpha} \kappa_{a b} \nu_{\beta}^{b}, \quad A_{i}^{\alpha}=\nu_{a}^{\alpha} A_{i}^{a}, \quad f_{\alpha \beta \gamma}=g_{0} \nu^{a}{ }_{\alpha} \nu^{b}{ }_{\beta} \nu^{c}{ }_{\gamma} \omega_{a b c},
$$

so that

$$
S_{\mathrm{Het}}=v \int d^{10} x \sqrt{g} e^{-2 \phi}\left[R+4 \partial_{i} \phi \partial^{i} \phi-\frac{1}{12} \hat{H}_{i j k} \hat{H}^{i j k}-\frac{1}{4} \kappa_{a b} F_{i j}^{a} F^{i j b}\right],
$$

with

$$
\begin{aligned}
\hat{H}_{i j k} & =3\left(\partial_{[i} B_{j k]}-\kappa_{a b} A_{[i}{ }^{a} \partial_{j} A_{k]}{ }^{b}\right)+g_{0} \kappa_{a d} \omega^{d}{ }_{b c} A_{[i}{ }^{a} A_{j}{ }^{b} A_{k]}{ }^{c}, \\
F_{i j}^{a} & =2 \partial_{[i} A_{j]}{ }^{a}+g_{0} \omega^{a}{ }_{b c} A_{[i}{ }^{b} A_{j]}{ }^{c} .
\end{aligned}
$$

\section{Conclusions}

We revisited the gauge symmetries of DFT, and derived necessary and sufficient consistency conditions for closure of gauge transformations, (2.9), (2.12) and gauge invariance of the action (2.15). These conditions select sets of fields and gauge parameters for which DFT is consistent. The only previously known solutions to these conditions obeyed the socalled weak and strong constraints (2.16), that restrict the possible configurations to be 
T-dualizable to a frame in which there is no dependence on dual coordinates. Here, we propose a configuration (3.2) à la Scherk-Schwarz, that should satisfy (3.27) and (3.26) for consistency, but not necessarily the weak or strong constraint.

On these configurations, DFT is effectively described by a lower dimensional Gauged DFT, a DFT-like theory deformed by gaugings that preserve the global covariance of the parent theory. The action (3.6), global symmetries, gauge symmetries (3.15), (3.16), bracket (3.18) and constraints (3.20), (3.22) and (3.25) are all derived from those of DFT (2.2), (2.3), (2.5), (2.7), (2.9), (2.12), (2.15).

Particular examples of GDFT are gauged supergravities and the non-Abelian heterotic supergravities. The embedding of the former into GDFT was discussed in [30, 31], and the later in [29]. We reviewed the results of [29] in the context of dimensional reductions, showing that the heterotic supergravities can be effectively obtained from Scherk-Schwarz compactifications of ungauged DFT.

There are a number of questions that follow from our analysis. In particular, it would be nice to find the classes of solutions to the constraints we found on the DFT, and determine if there is a maximal class. On the other hand, at the level of the reduced GDFT, it would be interesting to classify all the possible solutions to the constraints on the duality twists, and determine all the possible orbits of gaugings that can be turned on through this procedure. Generalizations of our procedure could allow for more general gaugings, like those obtained by turning on $f_{A}$. Another task would be to find an explicit example or existence proof of truly doubled backgrounds satisfying the constraints of section 3.3.

Finally, relaxing the strong constraint forces more ambitious questions upon us. Given that a background for which there is no frame where the dependence on the dual coordinates disappears, one cannot stay within a limit where higher string excitation modes are heavier than the modes kept here. In that case one wonders whether a formulation of DFT without the strong constraint really corresponds to some limit of string theory. ${ }^{2}$

\section{Acknowledgments}

We thank G. Aldazabal, D. Andriot, W. Baron, E. Bergshoeff, G. Dibitetto, D. Geissbuhler, A. Guarino, O. Hohm, K. Narain, C. Nuñez, D. Roest, A. Rosabal, D. Thompson, D. Waldram and B. Zwiebach for enlightening discussions and correspondence. This work was supported by the ERC Starting Independent Researcher Grant 259133 - ObservableString and by ECOS-Sud France binational collaboration project A08E06.

Open Access. This article is distributed under the terms of the Creative Commons Attribution License which permits any use, distribution and reproduction in any medium, provided the original author(s) and source are credited.

\section{References}

[1] C. Hull and B. Zwiebach, Double field theory, JHEP 09 (2009) 099 [arXiv:0904.4664] [INSPIRE].

\footnotetext{
${ }^{2}$ We thank D. Waldram and B. Zwiebach for sharing their viewpoints with us.
} 
[2] C. Hull and B. Zwiebach, The gauge algebra of double field theory and Courant brackets, JHEP 09 (2009) 090 [arXiv:0908.1792] [INSPIRE].

[3] O. Hohm, C. Hull and B. Zwiebach, Background independent action for double field theory, JHEP 07 (2010) 016 [arXiv: 1003.5027] [INSPIRE].

[4] O. Hohm, C. Hull and B. Zwiebach, Generalized metric formulation of double field theory, JHEP 08 (2010) 008 [arXiv: 1006.4823] [INSPIRE].

[5] W. Siegel, Superspace duality in low-energy superstrings, Phys. Rev. D 48 (1993) 2826 [hep-th/9305073] [INSPIRE].

[6] W. Siegel, Two vierbein formalism for string inspired axionic gravity, Phys. Rev. D 47 (1993) 5453 [hep-th/9302036] [INSPIRE].

[7] A.A. Tseytlin, Duality symmetric closed string theory and interacting chiral scalars, Nucl. Phys. B 350 (1991) 395 [inSPIRE].

[8] A.A. Tseytlin, Duality symmetric formulation of string world sheet dynamics, Phys. Lett. B 242 (1990) 163 [INSPIRE].

[9] M. Duff, Duality rotations in string theory, Nucl. Phys. B 335 (1990) 610 [InSPIRE].

[10] M. Duff and J. Lu, Duality rotations in membrane theory, Nucl. Phys. B 347 (1990) 394 [INSPIRE].

[11] O. Hohm and S.K. Kwak, $N=1$ supersymmetric double field theory, JHEP 03 (2012) 080 [arXiv: 1111.7293] [INSPIRE].

[12] I. Jeon, K. Lee and J.-H. Park, Supersymmetric double field theory: stringy reformulation of supergravity, Phys. Rev. D Rapid Communications 85, 081501 (R) (2012) [arXiv: 1112.0069] [INSPIRE].

[13] N.B. Copland, A double $\sigma$-model for double field theory, arXiv:1111.1828 [INSPIRE].

[14] D.S. Berman, E.T. Musaev and M.J. Perry, Boundary terms in generalized geometry and doubled field theory, Phys. Lett. B 706 (2011) 228 [arXiv:1110.3097] [INSPIRE].

[15] I. Jeon, K. Lee and J.-H. Park, Incorporation of fermions into double field theory, JHEP 11 (2011) 025 [arXiv:1109.2035] [INSPIRE].

[16] N. Kan, K. Kobayashi and K. Shiraishi, Equations of motion in double field theory: from particles to scale factors, Phys. Rev. D 84 (2011) 124049 [arXiv:1108.5795] [InSPIRE].

[17] O. Hohm, S.K. Kwak and B. Zwiebach, Double field theory of type II strings, JHEP 09 (2011) 013 [arXiv:1107.0008] [INSPIRE].

[18] O. Hohm, S.K. Kwak and B. Zwiebach, Unification of type II strings and T-duality, Phys. Rev. Lett. 107 (2011) 171603 [arXiv:1106.5452] [INSPIRE].

[19] N.B. Copland, Connecting T-duality invariant theories, Nucl. Phys. B 854 (2012) 575 [arXiv: 1106.1888] [INSPIRE].

[20] C. Albertsson, S.-H. Dai, P.-W. Kao and F.-L. Lin, Double field theory for double D-branes, JHEP 09 (2011) 025 [arXiv:1107.0876] [INSPIRE].

[21] D.C. Thompson, Duality invariance: from M-theory to double field theory, JHEP 08 (2011) 125 [arXiv:1106.4036] [INSPIRE].

[22] D. Andriot, M. Larfors, D. Lüst and P. Patalong, A ten-dimensional action for non-geometric fluxes, JHEP 09 (2011) 134 [arXiv:1106.4015] [INSPIRE].

[23] I. Jeon, K. Lee and J.-H. Park, Stringy differential geometry, beyond Riemann, Phys. Rev. D 84 (2011) 044022 [arXiv: 1105.6294] [INSPIRE]. 
[24] I. Jeon, K. Lee and J.-H. Park, Double field formulation of Yang-Mills theory, Phys. Lett. B 701 (2011) 260 [arXiv: 1102.0419] [INSPIRE].

[25] O. Hohm and S.K. Kwak, Frame-like geometry of double field theory, J. Phys. A 44 (2011) 085404 [arXiv:1011.4101] [InSPIRE].

[26] I. Jeon, K. Lee and J.-H. Park, Differential geometry with a projection: application to double field theory, JHEP 04 (2011) 014 [arXiv: 1011.1324] [INSPIRE].

[27] S.K. Kwak, Invariances and equations of motion in double field theory, JHEP 10 (2010) 047 [arXiv: 1008.2746] [INSPIRE].

[28] O. Hohm and S.K. Kwak, Massive type II in double field theory, JHEP 11 (2011) 086 [arXiv: 1108.4937] [INSPIRE].

[29] O. Hohm and S.K. Kwak, Double field theory formulation of heterotic strings, JHEP 06 (2011) 096 [arXiv:1103.2136] [INSPIRE].

[30] G. Aldazabal, W. Baron, D. Marques and C. Núñez, The effective action of double field theory, JHEP 11 (2011) 052 [Erratum ibid. 1111 (2011) 109] [arXiv:1109.0290] [INSPIRE].

[31] D. Geissbuhler, Double field theory and $N=4$ gauged supergravity, JHEP 11 (2011) 116 [arXiv: 1109.4280] [INSPIRE].

[32] O. Hohm and B. Zwiebach, On the Riemann tensor in double field theory, arXiv:1112.5296 [INSPIRE].

[33] A. Coimbra, C. Strickland-Constable and D. Waldram, Supergravity as generalised geometry I: type II theories, JHEP 11 (2011) 091 [arXiv:1107.1733] [INSPIRE].

[34] A. Coimbra, C. Strickland-Constable and D. Waldram, $E_{d(d)} \times \mathbb{R}^{+}$generalised geometry, connections and M-theory, arXiv:1112.3989 [INSPIRE].

[35] P. West, Generalised geometry, eleven dimensions and $E_{11}$, JHEP 02 (2012) 018 [arXiv:1111.1642] [INSPIRE].

[36] A. Rocen and P. West, $E_{11}$, generalised space-time and IIA string theory: the RR sector, arXiv: 1012.2744 [INSPIRE].

[37] P. West, $E_{11}$, generalised space-time and IIA string theory, Phys. Lett. B 696 (2011) 403 [arXiv: 1009.2624] [inSPIRE].

[38] D.S. Berman, H. Godazgar, M.J. Perry and P. West, Duality invariant actions and generalised geometry, JHEP 02 (2012) 108 [arXiv:1111.0459] [INSPIRE].

[39] D.S. Berman, H. Godazgar, M. Godazgar and M.J. Perry, The local symmetries of M-theory and their formulation in generalised geometry, JHEP 01 (2012) 012 [arXiv:1110.3930] [INSPIRE].

[40] D.S. Berman and M.J. Perry, Generalized geometry and M-theory, JHEP 06 (2011) 074 [arXiv: 1008.1763] [INSPIRE].

[41] O. Hohm, T-duality versus gauge symmetry, Prog. Theor. Phys. Suppl. 188 (2011) 116 [arXiv:1101.3484] [INSPIRE].

[42] B. Zwiebach, Double field theory, T-duality and Courant brackets, arXiv:1109.1782 [INSPIRE].

[43] J. Scherk and J.H. Schwarz, How to get masses from extra dimensions, Nucl. Phys. B 153 (1979) 61 [INSPIRE].

[44] J. Shelton, W. Taylor and B. Wecht, Nongeometric flux compactifications, JHEP 10 (2005) 085 [hep-th/0508133] [INSPIRE]. 
[45] G. Aldazabal, P.G. Camara, A. Font and L. Ibáñez, More dual fluxes and moduli fixing, JHEP 05 (2006) 070 [hep-th/0602089] [INSPIRE].

[46] G. Aldazabal, E. Andres, P.G. Camara and M. Graña, U-dual fluxes and generalized geometry, JHEP 11 (2010) 083 [arXiv: 1007.5509] [INSPIRE].

[47] C. Hull, A geometry for non-geometric string backgrounds, JHEP 10 (2005) 065 [hep-th/0406102] [INSPIRE].

[48] A. Dabholkar and C. Hull, Generalised T-duality and non-geometric backgrounds, JHEP 05 (2006) 009 [hep-th/0512005] [INSPIRE].

[49] C. Hull and R. Reid-Edwards, Gauge symmetry, T-duality and doubled geometry, JHEP 08 (2008) 043 [arXiv:0711.4818] [INSPIRE].

[50] C. Hull and R. Reid-Edwards, Non-geometric backgrounds, doubled geometry and generalised T-duality, JHEP 09 (2009) 014 [arXiv:0902.4032] [inSPIRE].

[51] G. Dall'Agata, N. Prezas, H. Samtleben and M. Trigiante, Gauged supergravities from twisted doubled tori and non-geometric string backgrounds, Nucl. Phys. B 799 (2008) 80 [arXiv: 0712.1026] [INSPIRE].

[52] H. Samtleben, Lectures on gauged supergravity and flux compactifications, Class. Quant. Grav. 25 (2008) 214002 [arXiv:0808.4076] [INSPIRE].

[53] D. Andriot, E. Goi, R. Minasian and M. Petrini, Supersymmetry breaking branes on solvmanifolds and de Sitter vacua in string theory, JHEP 05 (2011) 028 [arXiv:1003.3774] [INSPIRE].

[54] D. Andriot, R. Minasian and M. Petrini, Flux backgrounds from twists, JHEP 12 (2009) 028 [arXiv: 0903.0633] [INSPIRE].

[55] J. Schon and M. Weidner, Gauged N=4 supergravities, JHEP 05 (2006) 034 [hep-th/0602024] [INSPIRE].

[56] G. Aldazabal, D. Marques, C. Núñez and J.A. Rosabal, On type IIB moduli stabilization and $N=4,8$ supergravities, Nucl. Phys. B 849 (2011) 80 [arXiv:1101.5954] [INSPIRE].

[57] G. Dibitetto, A. Guarino and D. Roest, How to halve maximal supergravity, JHEP 06 (2011) 030 [arXiv: 1104.3587] [INSPIRE].

[58] M. Graña, R. Minasian, M. Petrini and D. Waldram, T-duality, generalized geometry and non-geometric backgrounds, JHEP 04 (2009) 075 [arXiv: 0807.4527] [INSPIRE].

[59] D.J. Gross, J.A. Harvey, E.J. Martinec and R. Rohm, Heterotic string theory. 2. The interacting heterotic string, Nucl. Phys. B 267 (1986) 75 [INSPIRE].

[60] D.J. Gross, J.A. Harvey, E.J. Martinec and R. Rohm, Heterotic string theory. 1. The free heterotic string, Nucl. Phys. B 256 (1985) 253 [InSPIRE].

[61] D. Andriot, Heterotic string from a higher dimensional perspective, Nucl. Phys. B 855 (2012) 222 [arXiv:1102.1434] [INSPIRE].

[62] J. Maharana and J.H. Schwarz, Noncompact symmetries in string theory, Nucl. Phys. B 390 (1993) 3 [hep-th/9207016] [INSPIRE].

[63] N. Kaloper and R.C. Myers, The odd story of massive supergravity, JHEP 05 (1999) 010 [hep-th/9901045] [INSPIRE]. 